\title{
The evaluation of fibrotic effects of the hepatitis B virus pre-core in hepatic stellate cells
}

\author{
SEYED YOUNES HOSSEINI ${ }^{1,2}$, KAZEM BAESI $^{3}$, NEGAR AZARPIRA $^{4}$, \\ AMENEH PAKNEIAT $^{2}$ and SEYEDEH AKRAM HOSSEINI ${ }^{2}$
}

\author{
${ }^{1}$ Department of Bacteriology and Virology, School of Medicine; ${ }^{2}$ Gastroenterohepatology Research Center, \\ Shiraz University of Medical Sciences, Shiraz 71348-45794; ${ }^{3}$ Hepatitis and AIDS Department, Pasteur institute of Iran, \\ Tehran 13169-43551; ${ }^{4}$ Transplant Research Center, Shiraz University of Medical Sciences, Shiraz 14336-71348, Iran
}

Received February 9, 2017; Accepted March 15, 2017

DOI: $10.3892 / b r .2017 .894$

\begin{abstract}
The role of the hepatitis B virus (HBV) endogenous pre-core protein in liver fibrosis is controversial. Whether the expression of the pre-core induces the activation of human stellate cells (HSCs) has not yet been reported. Plasmids expressing $\mathrm{HBx}$, or pre-core protein were transfected into LX-2 cells. Subsequently, total RNA extracted and reverse transcription-quantitative polymerase chain reaction was performed to measure the fold change of collagen type I, $\alpha 1$ chain, $\alpha$-smooth muscle actin and TIMP metalloproteinase inhibitor-1. Moreover, transforming growth factor (TGF)- $\beta$ in the supernatant of HSCs was evaluated by ELISA assay. In addition, a MTT assay was performed to test the cytotoxicity of the endogenous expression in LX-2 cells. None of the plasmids exhibited cytotoxic nor significant proliferative effects on LX-2 cells by MTT assessment. The gene expression analysis of fibrotic genes in LX-2 cells demonstrated that the pre-core protein presented no significant $(\mathrm{P}>0.05)$ fibrotic impact when compared to the empty control plasmid and HBx. The data from the TGF- $\beta$ ELISA was consistent with the mRNA expression as detected with the control plasmid $(\mathrm{P}>0.05)$. The endogenous expression of the HBV pre-core exhibited no fibrotic impression in HSCs when compared to HBx.
\end{abstract}

\section{Introduction}

Liver fibrosis that progresses by excessive deposition of the extracellular matrix, is a frequent consequence of chronic liver damage following hepatitis B virus (HBV) infection $(1,2)$. Among liver cells, human stellate cells (HSCs) serve a key role in the development of liver fibrosis (2). While the molecular mechanism of fibrosis induction by hepatitis $\mathrm{C}$ virus (HCV)

Correspondence to: Dr Seyed Younes Hosseini, Department of Bacteriology and Virology, School of Medicine, Shiraz University of Medical Sciences, Shiraz 71348-45794, Iran

E-mail: hoseiniy@sums.ac.ir

Key words: fibrosis, hepatitis B virus, pre-core protein, hepatic stellate cell is well described and is mostly attributed to core and NS3 proteins (3), the responsible HBV proteins and underlying mechanisms remain obscure. The direct influence of HBV proteins in the fibrosis process requires further investigation (4).

In spite of some reports concerning fibrotic roles of $\mathrm{HCV}$ core protein (5-8), the impact of the HBV core protein (HBc) on HSCs is described poorly. The HBc is the primary structural unit of the capsid, consisting of 185 amino acids. The carboxyl terminal region (aa 150-185) has previously been demonstrated to interact closely with the viral RNA pre-genome or DNA genome (9). The HBV core protein has been identified to exhibit a tendency for inflammation in the liver site, with previous studies demonstrating that truncation of the core by removing this carboxyl end reduces inflammation (10-12).

The research concerning the effect of the HBc protein on fibrosis is limited. Sequencing data using patients sample suggested that core protein mutations of the $\mathrm{C}$-terminal domain contribute to the stage of fibrosis due to functional alterations (13). The protein structure of core and HBe proteins are homologous. The exception is the pre-core sequence in $\mathrm{HBe}$, which helps protein to be secreted from host cells. A recent study stated that $\mathrm{HBe}$ gene exhibited fibrosis when transfected into a rat stellate cell line (14).

As hepatic stellate cells demonstrated to be a transient supportive site of HBV infection/replication in vitro (4) it is noticeable if endogenous pre-core expression in human HSCs establish a fibrosis process or not. Several previous studies provided direct evidence that HBV could replicate and express structural proteins in HSCs cells (4). As plausible mechanism of fibrosis, mechanism behind endogenous expression of core and related derivatives may clarify molecular paths for fibrosis as well as new targets. Furthermore, the exact role pre-core protein needed to be investigated.

\section{Materials and methods}

Cell culture and plasmids transfection. The immortalized human stellate cell line, LX-2, was provided by Professor Scott Friedman (Mount Sinai School of Medicine, New York City, NY, USA). It displays typical features of partially active stellate cells that can be employed as a sustained model for HSC research (15). The cells were cultured and maintained on 
$25 \mathrm{~cm}^{2}$ plastic tissue culture flasks in low glucose Dulbecco's modified Eagle's medium (DMEM; Gibco; Thermo Fisher Scientific, Inc., Waltham, MA, USA) supplemented with $2 \mathrm{mM}$ L-glutamine, $100 \mathrm{U} / \mathrm{ml}$ penicillin-streptomycin (Gibco; Thermo Fisher Scientific, Inc.), 5\% fetal bovine serum (Gibco; Thermo Fisher Scientific, Inc.) and incubated at $37^{\circ} \mathrm{C}$ in $5 \%$ $\mathrm{CO}_{2}$ air humidified atmosphere.

The pCAGGS plasmids expressing pre-core and $\mathrm{HBxAg}$ proteins was kindly provided by Dr. Gloria Gonzalez-Aseguinolaza [Centro de Investigación Médica Aplicada (CIMA), Universidad de Navarra, Pamplona, Spain] and were named pCAG-pre-core and pCAG-X, respectively. The expression analysis of both plasmids has previously been performed at this center by western blot analysis or reverse transcription-quantitative polymerase chain reaction (RT-qPCR) assays (unpublished data).

LX- 2 cells $\left(4 \times 10^{5}\right.$ cells) were seeded in six-well plate and, following $24 \mathrm{~h}$, when it reached $80 \%$ confluence, they were transfected with $1 \mu \mathrm{g}$ DNA plasmid using Lipofectamine $2000^{\mathrm{TM}}$ reagent (Invitrogen; Thermo Fisher Scientific, Inc.) in serum free DMEM medium, according to the manufacturer's instructions. At $6 \mathrm{~h}$ following transfection, the medium was changed with fresh complete DMEM medium and the plate was left for further $18 \mathrm{~h}$. A group of cells (equal number to test groups) was treated by $75 \mathrm{ng} / \mathrm{ml}$ human leptin, a pro-fibrotic hormone (Sigma-Aldrich; Merck KGaA, Darmstadt, Germany), was used as a positive control. Hence, cells that were transfected by empty pCAG plasmid in a separate well enrolled as negative control group and designated as the 'plasmid group'.

Cell proliferation assay. The MTT assay was performed to determine the effect of different components on activated LX-2 cell proliferation as described before (16). In brief, $7.5 \times 10^{3}$ cells were seeded into 96-well plates, before being transfected by Lipofectamine reagent. The cells were incubated at $37^{\circ} \mathrm{C}$ in humidified $\mathrm{CO}_{2}$ incubator for $48 \mathrm{~h}$. At 2 days following incubation, supernatants were changed to fresh medium and $10 \mu 1$ MTT (Sigma-Aldrich; Merck KGaA) at a concentration of $5 \mathrm{mg} / \mathrm{ml}$. Dimethylsulfoxide (CinnaGen, Inc., Tehran, Iran) was added to each well and absorbance was determined by dual wavelengths of 570 and $630 \mathrm{~nm}$ on a microplate ELISA reader (BioTek Elx 808; BioTek Instruments, Inc., Winooski, VT, USA). Percentage inhibition of proliferation was calculated as follows: $\%$ inhibition $=100-[$ (absorbance of test/absorbance of control) x 100].

RNA extraction and reverse transcription. Total RNA was extracted from treated cells $36 \mathrm{~h}$ post TGF- $\beta$ activation, with the help of TRIzol reagent (Invitrogen; Thermo Fisher Scientific, Inc.) according to manufacturer's protocol. Total RNA was treated with RQ1 DNase (Promega Corporation, Madison, WI, USA) to avoid residual DNA contamination. The cDNA synthesis was made by RocketScript RT Premix kit (Bioneer Corporation, Daejon, Korea) based on manufacturer's protocol. The prepared cDNA was stored at $-20^{\circ} \mathrm{C}$ condition until use.

$R T$-qPCR expression analysis. The primer pairs for $\alpha$-smooth muscle actin ( $\alpha$-SMA), collagen type I, $\alpha 1$ chain (COL1A1),
TIMP metalloproteinase inhibitor 1 (TIMP-1) and GAPDH quantification were described and listed previously (17). The GAPDH gene was used as reference of normalization in all samples while $\mathrm{H}_{2} \mathrm{O}$ as a negative control and RNA mixture were included in each run. The mRNA levels were relatively quantified using SYBR-Green I master mix (Takara Bio, Inc., Otsu, Japan) by the help of StepOnePlus Real-Time PCR system (Applied Biosystems; Thermo Fisher Scientific, Inc.). The reaction mixtures prepared according to recommended protocol and amplification reaction was performed in 40 cycles of $10 \mathrm{sec}$ at $94^{\circ} \mathrm{C}$ for denaturation and $25 \mathrm{sec}$ at $58^{\circ} \mathrm{C}$ for the annealing step. The threshold value for each sample was normalized to the GAPDH signal, and then the $2^{-\Delta \Delta C q}$ equation was used for relative expression assay of mRNAs, which finally was presented as fold-change (18).

ELISA assay for TGF- $\beta 1$ measurement. At $\sim 36 \mathrm{~h}$ following LX- 2 cell activation with TGF- $\beta$, released bioactive TGF- $\beta 1$ in supernatant was measured by the help of the Human-Mouse TGF- $\beta$ ELISA kit (eBioscience, Inc.; Thermo Fisher Scientific, Inc.) according to the manufacturer's instruction. All the samples were first acid-activated before starting the method and background TGF- $\beta 1$ level (due to adding TGF- $\beta$ for HSC activation) was deleted from final analysis. The absorbance was measured at $450 \mathrm{~nm}$ by ELISA reader (BioTek Instruments, Inc.; Elx 808) and then data was calculated against standard curve and adjusted to $\mathrm{pg} / \mathrm{ml}$ of culture medium.

Statistical analysis. Statistical analysis was conducting using GraphPad Prism (version 5; GraphPad Prism Software, Inc., La Jolla, CA, USA) that used a one-way analysis of variance to evaluate the differences between means. The statistical significance between controls and test groups was evaluated by Tukey's post hoc test and $\mathrm{P}<0.05$ was considered to indicate a statistically significant difference.

\section{Results}

No significant proliferation was induced by the pre-core gene in LX-2 cells. To compare the anti-proliferative effects of plasmid expression, LX2 cell viability was measured by MTT assay. Whereas the proliferation inhibitory impact of all constructs was detectable on activated HSCs, the significant impact was not detectable for all constructs. (Data not shown).

Pre-core sequence induced a negligible fibrotic effect. The mRNA expression levels for pro-fibrotic genes including TIMP-1, COL1A1, $\alpha$-SMA were quantified by RT-qPCR following exposure. As presented in Fig. 1, activated LX-2 (the cells incubated with leptin as positive control) displayed an increase in production of the extracellular matrix including $\alpha$-SMA, COL1A1 and TIMP-1 by 4.3-, 3.9- and 3.1-fold, respectively. Hence, it was demonstrated that the HBX gene, another HBV protein, also exhibited a significant fibrotic impact $(\mathrm{P}<0.01)$ by 2.2-, 2.3- and 3-fold compared to control plasmid group or untreated cells. In contrast, the pre-core protein exhibited no significant effect with an expression upregulation of 1.3, 1.2 and 0.9 for a-SMA, collagen type 1 and TIMP-1, respectively (all P>0.05) when compared to both plasmid control group and untreated cell group, as depicted in Fig. 1. 

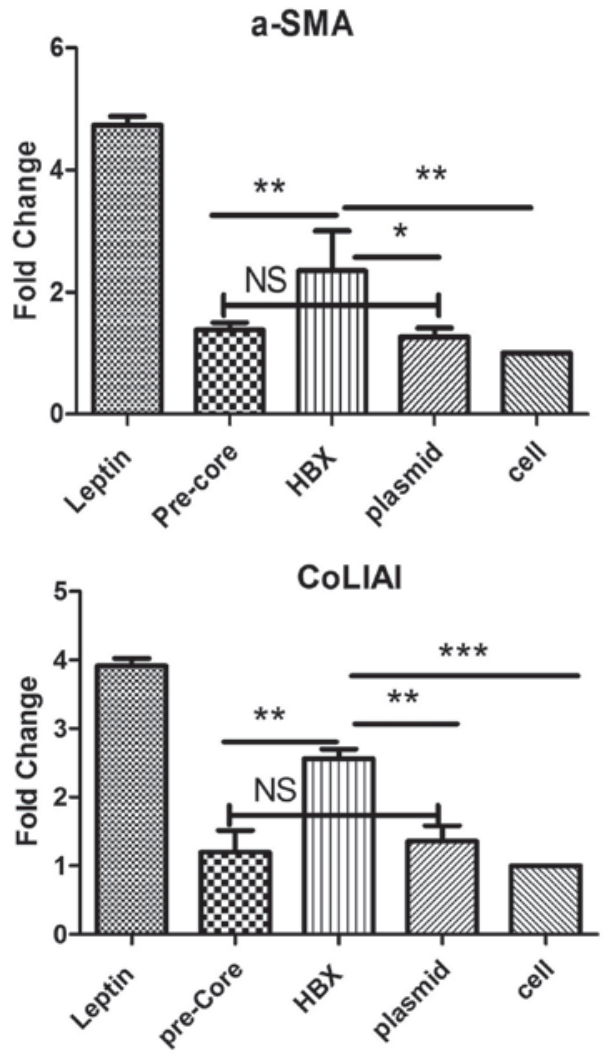

TIMP-1

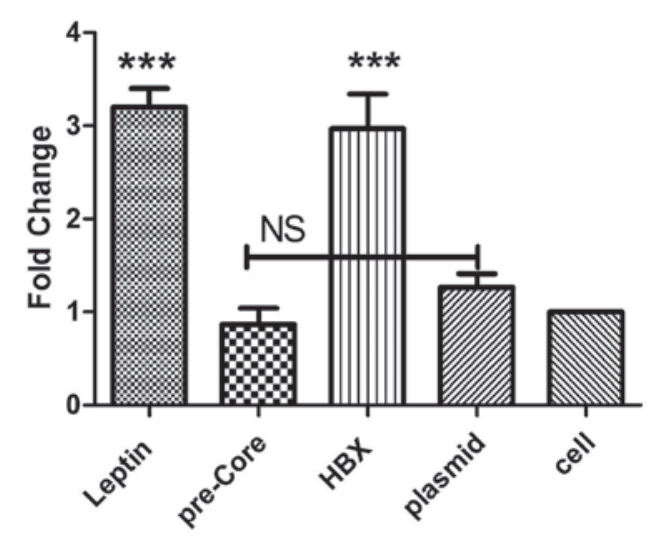

Figure 1. The results of gene expression analysis in LX-2 cells, investigating (A) $\alpha$-SMA, (B) COL1A1 and (C) TIMP-1. The pre-core sequence exhibited no significant change in expression levels of fibrotic molecules in LX-2 cells, when compared with the plasmid control and untreated cell groups. HBx significantly presented its fibrotic role, as expression of pro-fibrotic molecules increased when compared with other groups, such as pre-core group $(\mathrm{P}<0.01)$. Each bar is representative of the mean \pm standard deviation for at least 3 measurements and the result presents the fold increase/decrease. ${ }^{*} \mathrm{P}<0.05,{ }^{* *} \mathrm{P}<0.01$ and ${ }^{* * *} \mathrm{P}<0.001 . \alpha$-SMA, $\alpha$-smooth muscle actin; COL1A1, collagen type I, $\alpha 1$ chain; TIMP-1, TIMP metalloproteinase inhibitor-1; NS, not significant.

Pre-core sequence did not up-regulate TGF- $\beta 1$ production. Following plasmid transfection, TGF- $\beta 1$ cytokine production was evaluated in culture supernatants by ELISA assay (Fig. 2). The result indicated that, with the exception of leptin treatment, all constructs left no significant impact on TGF- $\beta 1$ production on LX-2. In addition, the data indicated that the cell with no treatment also produced a measurable level of TGF- $\beta 1(230 \mathrm{pg} / \mathrm{ml})$.

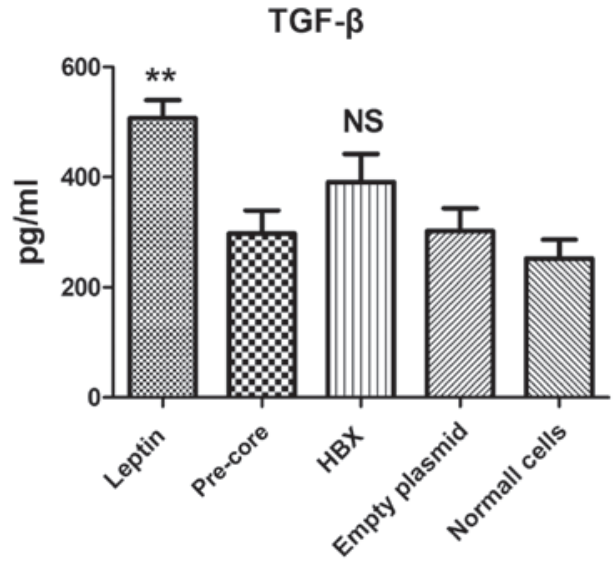

Figure 2. Effects of different hepatitis B virus genes on TGF- $\beta$ production. In spite of the leptin positive group that significantly induced cytokine production, the HBX and pre-core groups presented no significant difference to any of the negative control groups. ${ }^{* *} \mathrm{P}<0.01$ vs. all other groups. Normal cells (negative untreated control). Each bar is representative of the mean \pm standard deviation. TGF- $\beta$, transforming growth factor- $\beta$; NS, not significant.

\section{Discussion}

Fibrosis is an outcome of elongated liver damage, arising from HBV chronic infection (19). Similarly, prolonged infection of HBV in a mouse model may consequently develop fibrosis in liver (20). Hepatic HSCs accounted as the most important components in the establishment and progression of fibrosis. The alteration of their gene expression patterns are responsible for extracellular matrix subversion, which ultimately leads to fibrosis progression $(21,22)$. The molecular mechanism of fibrosis by $\mathrm{HBV}$ is not clearly clarified and the direct role of corresponding viral proteins in the process of fibrosis is also debated (23). Therefore, the study of the interaction of HBV proteins with this lineage of cells may facilitate the understanding of causes and give new opportunity to take control of it.

In one study, HBV-like particles were identified in the LX-2 cellular cytoplasm with electron microscopy and, while HSCs were permissive for virus replication, productive replication of HBV does not occur (4). Regarding the transient supportive role of HSCs in HBV replication (4), it would be interesting to understand whether endogenous pre-core expression in human HSCs establishes fibrosis. A previous study provided direct evidence that HBV could replicate and express structural proteins in HSCs cells (4). In spite of different reports concerning the profound role of $\mathrm{HCV}$ core protein in liver fibrosis, the impact of HBV core protein remains to be elucidated in HSCs (5-8).

Some previous studies demonstrated the definitive fibrotic role of the HBX protein on HSCs in vitro (24-26). However, the fibrotic role of other HBV proteins is not understood and requires more investigation. The core protein presented an inflammatory action in the liver, therefore it may be predicted that it serves a role in the fibrosis process. Furthermore, previous works demonstrated that truncation of the core from the carboxyl end reduces inflammatory property of protein $(10,27)$. The carboxyl terminal region (aa 150-185) interacts closely with the viral RNA pre-genome or the viral DNA genome and regulates virus replication (9). Sequencing data also suggests that core protein mutations of $\mathrm{C}$-terminal domain 
contribute to further progression of liver fibrosis due to function alteration (13). A recent study by Zan et al (14) claimed that the HBe protein, the homologous antigen of core protein exhibited a fibrotic role in vitro. To the best of the authors' knowledge, no reports about the pre-core gene have been published thus far.

The present study demonstrated that, similar to previous efforts, HBX upregulates $\alpha$-SMA, COLIA1 and TIMP- 1 expression to a level comparable with leptin, a fibrosis inducer (24-26). Those reports employed HBX protein from a transfected hepatocyte supernatant, however, the current study investigated the endogenous HBX expression effect on fibrosis genes following direct HSC transfection.

The primary outcome of the paper involved the investigation into the possible effect of pre-core on LX-2 cell fibrotic gene expression. Assessment of endogenous expression of the HBV pre-core protein in HSCs and evaluation of subsequent fibrosis has not been researched prior. Some methodological limitations should be considered when interpreting these results. For example, the authors could not analyze protein expression or relevant secreted forms from supernatant.

These data indicated that the pre-core protein presented a negligible fibrotic property when compared to the control plasmid. In comparison to normal LX-2 cells, transfected cells using different plasmids demonstrated little fibrotic effect due to DNA structure. Gene expression analysis of COL1A1, $\alpha$-SMA and TIMP-1 demonstrated that expression of the pre-core protein exhibited a similar pattern of fibrosis to control groups. The authors conclude that the pre-core gene does not perform a fibrotic role during the fibrosis process of HBV. However, to further confirm of these in vitro achievements, more investigation in a suitable animal model is required.

\section{Acknowledgements}

The authors would like to thank the Laboratory staff of the Gastroenterohepatology Research Center, Shiraz University of Medical Sciences. The current study was funded by Shiraz University of Medical Sciences (grant no. 94-01-01-9968).

\section{References}

1. Gutierrez-Reyes G, Gutierrez-Ruiz MC and Kershenobich D: Liver fibrosis and chronic viral hepatitis. Arch Med Res 38: 644-651, 2007.

2. Mormone E, George $\mathrm{J}$ and Nieto N: Molecular pathogenesis of hepatic fibrosis and current therapeutic approaches. Chem Biol Interact 193: 225-231, 2011.

3. Khanizadeh S, Ravanshad M, Hosseini SY, Davoodian P, Zadeh AN, Sabahi F, Sarvari J, Khanlari Z and Hasani-Azad M: The possible role of NS3 protease activity of hepatitis $C$ virus on fibrogenesis and miR-122 expression in hepatic stellate cells. Acta Virol 60: 242-248, 2016.

4. Liu X, Zhu ST, You H, Cong M, Liu TH, Wang BE and Jia JD: Hepatitis B virus infects hepatic stellate cells and affects their proliferation and expression of collagen type I. Chin Med J (Engl) 122: 1455-1461, 2009.

5. Bataller R, Paik YH, Lindquist JN, Lemasters JJ and Brenner DA: Hepatitis C virus core and nonstructural proteins induce fibrogenic effects in hepatic stellate cells. Gastroenterology 126 : 529-540, 2004.

6. Coenen M, Nischalke HD, Krämer B, Langhans B, Glässner A, Schulte D, Körner C, Sauerbruch T, Nattermann J and Spengler U: Hepatitis $C$ virus core protein induces fibrogenic actions of hepatic stellate cells via toll-like receptor 2. Lab Invest 91: 1375-1382, 2011.
7. Shin JY, Hur W, Wang JS, Jang JW, Kim CW, Bae SH, Jang SK, Yang SH, Sung YC, Kwon OJ, et al: HCV core protein promotes liver fibrogenesis via up-regulation of CTGF with TGF-beta1. Exp Mol Med 37: 138-145, 2005.

8. Wu CF, Lin YL and Huang YT: Hepatitis C virus core protein stimulates fibrogenesis in hepatic stellate cells involving the obese receptor. J Cell Biochem, 2012.

9. Roseman AM, Berriman JA, Wynne SA, Butler PJ and Crowther RA: A structural model for maturation of the hepatitis B virus core. Proc Natl Acad Sci USA 102: 15821-15826, 2005.

10. Lee BO, Tucker A, Frelin L, Sallberg M, Jones J, Peters C, Hughes J, Whitacre D, Darsow B, Peterson DL and Milich DR: Interaction of the hepatitis B core antigen and the innate immune system. J Immunol 182: 6670-6681, 2009.

11. Manigold T, Böcker U, Chen J, Gundt J, Traber P, Singer MV and Rossol S: Hepatitis B core antigen is a potent inductor of interleukin-18 in peripheral blood mononuclear cells of healthy controls and patients with hepatitis B infection. J Med Virol 71: 31-40, 2003.

12. Vanlandschoot P, Van Houtte F, Serruys B and Leroux-Roels G: The arginine-rich carboxy-terminal domain of the hepatitis $B$ virus core protein mediates attachment of nucleocapsids to cell-surface-expressed heparan sulfate. J Gen Virol 86: 75-84, 2005.

13. Mohamadkhani A, Jazii FR, Poustchi H, Nouraein O, Abbasi S, Sotoudeh $\mathrm{M}$ and Montazeri G: The role of mutations in core protein of hepatitis B virus in liver fibrosis. Virol J 6: 209, 2009.

14. Zan Y, Zhang Y and Tien P: Hepatitis B virus e antigen induces activation of rat hepatic stellate cells. Biochem Biophys Res Commun 435: 391-396, 2013.

15. Xu L, Hui AY, Albanis E, Arthur MJ, O'Byrne SM, Blaner WS, Mukherjee P, Friedman SL and Eng FJ: Human hepatic stellate cell lines, LX-1 and LX-2: New tools for analysis of hepatic fibrosis. Gut 54: 142-151, 2005.

16. Hosseini SY, Kalantar K, Shahin K, Ghayour M, Rajabibazl M, Fattahi MR, et al: Comparison of the In Vitro Antifibrogenic

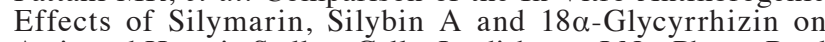
Activated Hepatic Stellate Cells. Jundishapur J Nat Pharm Prod Inpress: e40285, 2017.

17. Khanizadeh S, Ravanshad M, Hosseini S, Davoodian P, Nejati Zadeh A and Sarvari J: Blocking of SMAD4 expression by shRNA effectively inhibits fibrogenesis of human hepatic stellate cells. Gastroenterol Hepatol Bed Bench 8: 262-269, 2015.

18. Livak KJ and Schmittgen TD: Analysis of relative gene expression data using real-time quantitative PCR and the 2(-Delta Delta C(T)) Method. Methods 25: 402-408, 2001.

19. Guo XL, Liang B, Wang XW, Fan FG, Jin J, Lan R, et al: Glycyrrhizic acid attenuates CCl4-induced hepatocyte apoptosis in rats via a p53-mediated pathway. World journal of gastroenterology. World J Gastroenterol 19: 3781, 2013.

20. Ye L, Yu H, Li C, Hirsch ML, Zhang L, Samulski RJ, Li W and Liu Z: Adeno-Associated Virus Vector Mediated Delivery of the HBV Genome Induces Chronic Hepatitis B Virus Infection and Liver Fibrosis in Mice. PLoS One 10: e0130052, 2015.

21. Reeves HL and Friedman SL: Activation of hepatic stellate cells - a key issue in liver fibrosis. Front Biosci 7: d808-d826, 2002.

22. Kisseleva T and Brenner DA: Hepatic stellate cells and the reversal of fibrosis. J Gastroenterol Hepatol 21 (Suppl 3): S84-S87, 2006.

23. Lin J, Wu JF, Zhang Q, Zhang HW and Cao GW: Virus-related liver cirrhosis: Molecular basis and therapeutic options. World J Gastroenterol 20: 6457-6469, 2014.

24. Martín-Vílchez S, Sanz-Cameno P, Rodríguez-Muñoz Y, Majano PL, Molina-Jiménez F, López-Cabrera M, Moreno-Otero R and Lara-Pezzi E: The hepatitis B virus X protein induces paracrine activation of human hepatic stellate cells. Hepatology 47: 1872-1883, 2008.

25. Guo GH, Tan DM, Zhu PA and Liu F: Hepatitis B virus X protein promotes proliferation and upregulates TGF-betal and CTGF in human hepatic stellate cell line, LX-2. Hepatobiliary Pancreat Dis Int 8: 59-64, 2009.

26. Chen HY, Chen ZX, Huang RF, Lin N and Wang XZ: Hepatitis $B$ virus $X$ protein activates human hepatic stellate cells through upregulating TGFbeta1. Genetics and molecular research. GMR 13: 8645-8656, 2014.

27. Wu JF, Ni YH, Chen HL, Hsu HY and Chang MH: The impact of hepatitis B virus precore/core gene carboxyl terminal mutations on viral biosynthesis and the host immune response. J Infect Dis 209: 1374-1381, 2014. 